ex Instituto Archaeologico Universitatis de Rolando Eötvös nominatae

C

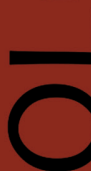

ப

$\varangle$

工

$\cup$

ه

$<$

in

ㄴ
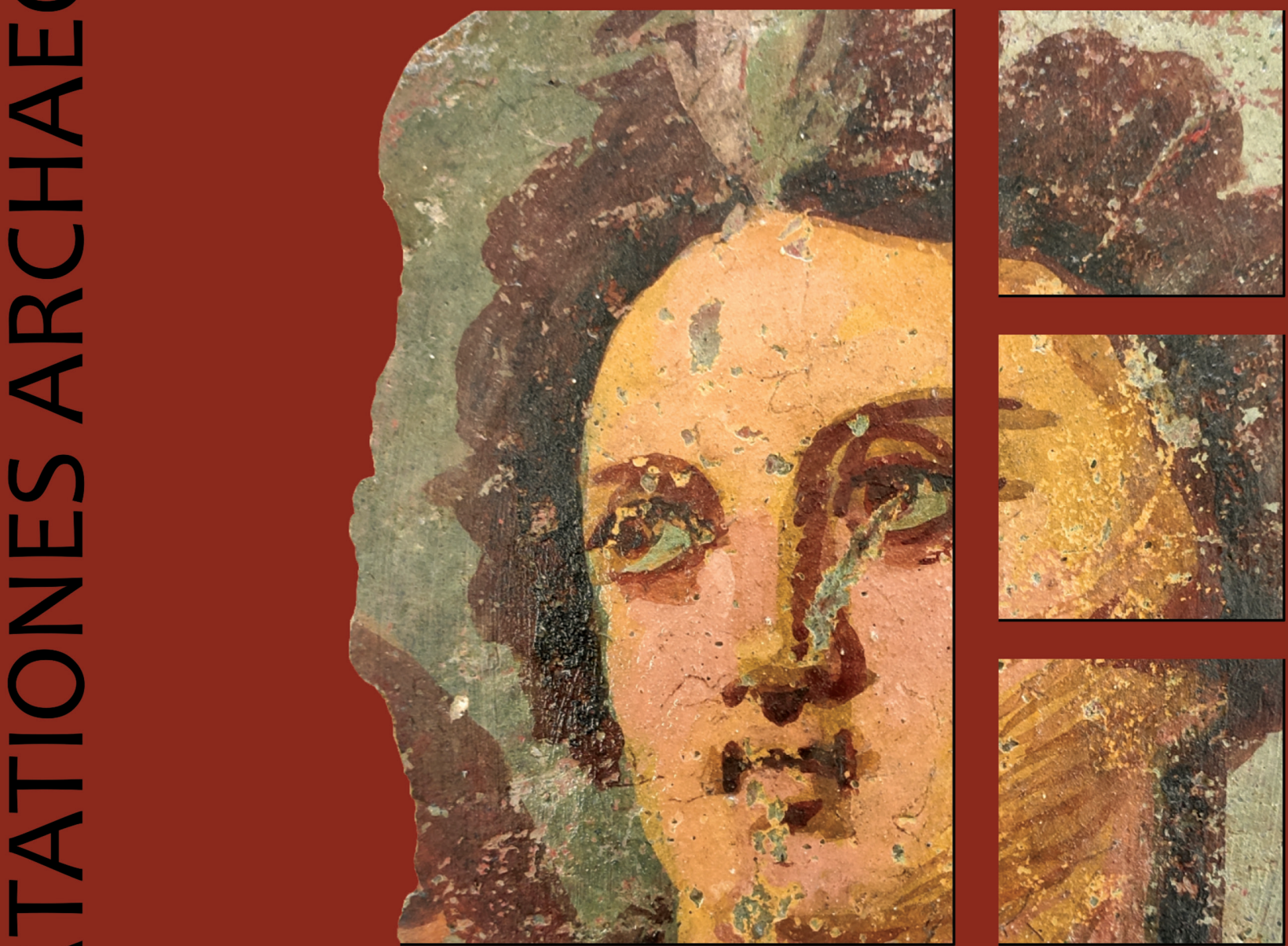

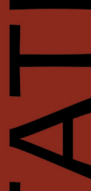

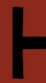

ח

Ш

un

n

0
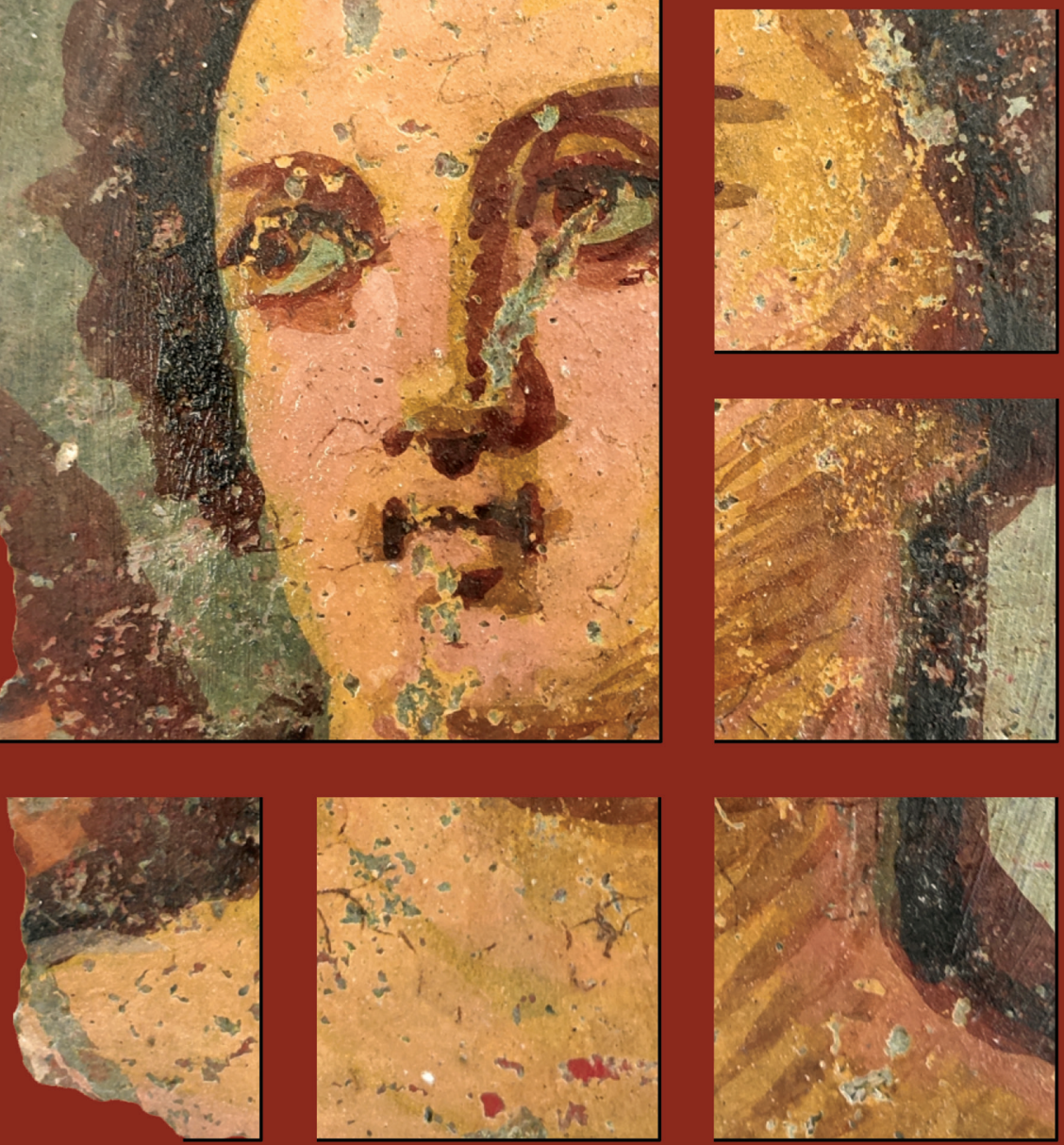

Serp 30 No. 8. 2020 


\section{Dissertationes Archaeologicae ex Instituto Archaeologico}

Universitatis de Rolando Eötvös nominatae Ser. 3. No. 8.

Budapest 2020 
Dissertationes Archaeologicae ex Instituto Archaeologico Universitatis de Rolando Eötvös nominatae Ser. 3. No. 8.

Editor-in-chief:

DÁvid Bartus

Editorial board:

LÁsZló BARTOSIEWICZ

LÁsZLÓ BORHY

ZOLTÁN CZAJLIK

IsTVÁN FELD

GÁBOR KALLA

PÁL RACZKY

MiKLÓS SZABÓ

Tivadar Vida

Technical editor:

Gábor VÁczi

Proofreading:

Szilvia BARTUS-SzÖLLŐsI

Zsófia KondÉ

Márton SZILÁGYI

Aviable online at http://ojs.elte.hu/dissarch

Contact: dissarch@btk.elte.hu

ISSN 2064-4574

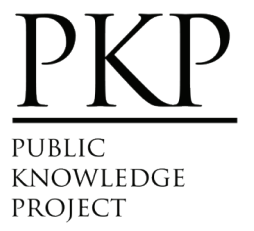

๑ ELTE Eötvös Loránd University, Institute of Archaeological Sciences

Layout and cover design: Gábor Váczi

Budapest 2020 


\section{CONTENTS}

\section{ARTICLES}

Maciej WAWRZCZAK - Zuzana KASENČÁKovÁ

Stará L'ubovña - Lesopark. Late Palaeolithic site and the problems associated with raw material mining

Attila PÉNTEK - Norbert FARAgó

Chipped stone assemblages from Schleswig-Holstein (North Germany) in the collection of the Institute of Archaeological Sciences - ELTE Eötvös Loránd University

Bence Soós 49

Middle Iron Age Cemetery from Alsónyék, Hungary

Tamás Szeniczey - Tamás Hajdu 107

Appendix - Results of the analysis of the Early Iron Age human remains unearthed at Alsónyék, Hungary

Lajos JuHÁsz - József Géza Kiss

Bound in bronze - a Roman bronze statuette of a barbarian prisoner

Csilla SÁRó

The fibula production of Brigetio: clay moulds

\section{Field Reports}

András Füzesi - Knut Rassmann - Eszter BÁnffy - Hajo Hoehler-Brockmann -

Gábor Kalla - Nóra Szabó - Márton SzIlágyi - Pál Raczky

Test excavation of the "pseudo-ditch" system of the Late Neolithic settlement complex at Öcsöd-Kováshalom on the Great Hungarian Plain

Gábor VÁczi - László RupNIK - Zoltán CZAJLIK - Gábor MEsterházy Bettina BitTner - Kristóf FÜlöP - Denisa M. LÖNHARdT - Nóra Szabó

The results of a non-destructive site exploration and a rescue excavation at the site of Pusztaszabolcs-Dohányos völgy északi part

Dávid BArtus - László Borhy - Szilvia JohÁczi - Emese SzÁmadó 181

Excavations in the legionary fortress of Brigetio in 2019 
Dávid BArtus - László Borhy - Emese SzÁmadó - Lajos Juhász - Bence Simon -

Ferenc Barna - Anita Benes - Szilvia Joháczi - Rita Olasz - Melinda Szabó

Excavations in Brigetio in 2020

\section{Thesis Abstracts}

Anett OszTÁs

The settlement history of Alsónyék-Bátaszék.

Complex analysis of its buildings in the context of the Lengyel culture

Csilla SzÁRAz

The region of the Zala and Mura Rivers (Zala County) in the Late Bronze Age.

Late Tumulus and Urnfield period

Ágnes KIRÁly

Human remains unearthed in settlement context from the Late Bronze Age -

Early Iron Age (Reinecke BD-HaB3) Northeastern Hungary

Gergely BóKA

Transformation of settlement history in the Körös Region in the period between the Late Bronze Age and the end of Iron Age

Gabriella G. DeLbó

Pottery production of the settlement complex of Brigetio

Adrienn Katalin BLAY

Die Beziehungen zwischen dem Karpatenbecken und dem Mediterraneum

von der II. Hälfte des 6. bis zum 8. Jahrhundert n. Chr. anhand Schmuckstücken

und Kleidungszubehör

Levente SAMU

293

Die mediterranen Kontakte des Karpatenbeckens in der Früh- und Mittel-

awarenzeit im Licht der Männerkleidung. Gürtelschnallen und Gürtelgarnituren

\section{REviEWS}

Gábor MESTERHÁZY

Czajlik, Z. - Črešnar, M. - Doneus, M. - Fera, M. - Hellmith Kramberger, A. Mele, M. (eds): Researching Archaelogical Landscapes Across Borders - Strategies,

Methods and Decisions for the 21th Century. Graz-Budapest, 2019. 


\title{
Die Beziehungen zwischen dem Karpatenbecken und dem Mediterraneum von der II. Hälfte \\ des 6. bis zum 8. Jahrhundert $\mathrm{n}$. Chr. anhand Schmuckstücken und Kleidungszubehör
}

\author{
Adrienn Katalin BLAY \\ blayadri@gmail.com
}

\begin{abstract}
Abstract of PhD thesis submitted in 2020 to the Archaeology Doctoral Programme, Doctoral School of History, Eötvös Loránd University under the supervision of Tivadar Vida, Budapest and to the Institute of the Prehistory and Medieval Archaeology at the Albert-Ludwigs-University of Freiburg (Breisgau) under the supervision of Sebastian Brather.
\end{abstract}

Das Byzantinische Reich und breiter die ganze mediterrane Welt übte bestimmt einen gewissen Einfluss auf ihren Randgebieten, was das Geistesleben, die Gebräuche und das allgemeine Leben der unterschiedlichen Schichten der awarenzeitlichen Gesellschaft erreichte. Durch ausgewählte Fundgruppen wurden es in der Dissertation dargestellt, inwieweit die mediterranen bzw. byzantinischen Einflüsse in der awarenzeitlichen materiellen Hinterlassenschaft erschienen wurden und sich widerspiegelt haben. Die Möglichkeiten für die Darstellung des kompletten Spektrums der mediterranen Kontakte der Bevölkerung sind aber ziemlich begrenzt, weil das hintergebliebene Material ursprünglich ,selektiert' ist. Die organischen Materialen - wie z.B. die Textilien, die Holzgegenstände - stehen nur in ganz außergewöhnlichen Fällen zur Verfügung. Die anorganischen Funde, wie die Metalle, die Knochengegenstände, die Keramik ${ }^{1}$ und die Gläser ${ }^{2}$ bieten ein breiteres Feld für die Forschung. Da aus Metall zahlreiche Gegenstandstypen - Geräte, ${ }^{3}$ Gefäße, ${ }^{4}$ Militaria, ${ }^{5}$ Kleidungszubehör, ${ }^{6}$ Schmuckstücke ${ }^{7}$ usw. - hergestellt wurden, wurden aus diesem Materialfeld hauptsächlich einige Gruppen für die Analyse ausgewählt: die Schmuckstücke und sonstige Kleidungszubehör, das wird aber mit anderen kleineren Fundgruppen (Gefäße - metallene sowie gläserne, Möbel, Gewichte, usw.) ergänzend analysiert, um ein komplexeres Bild zu kommen.

1 Mit den weiterlebenden spätantiken Traditionen in der Keramikherstellung hat sich ausführlich Tivadar Vida, in Bezug auf einem Fundort (Siedlung Kölked) hat sich Zsuzsa Hajnal auseinandergesetzt: VIDA 1999, 88-106; HajnAL 2005, 437-481. Mit den awarenzeitlichen Amphoren und mit ihren möglichen Verteilung haben sich letztlich Gergely Csiky und Piroska Magyar-Hárshegyi beschäftigt: CsIKY - MAGYAR-HÁRSHEGYI 2015, 175-182.

2 GARAm 2001, 169-172.

3 Mit den Waagen und Gewichten „byzantinischer” Herkunft haben sich ausführlich Zsófia Rácz und Tobias Bendeguz beschäftigt: ToBiAs 2008; RÁcz 2014, 100-106. Die awarenzeitlichen Schmiedewerkzeuge hat Zsófia Rácz bearbeitet: RÁcz 2014.

4 Die Metallgefäße wurden letztlich ausführlich in Bezug auf der Bronzekanne in Budakalász von Tivadar Vida erörtert: VIDA 2017.

5 Csiky 2015. Gergely Csiky hat die Stoß- und Schneidwaffen bearbeitet.

6 VIDA 2009, 233-260; VIDA 2018. Die Schnallen und Gürtelgarnituren werden von Levente Samu im Rahmen seiner Dissertation zusammengefasst.

7 Garam 2001, 15-87; Vida 2009, 240-241, Fig. 1; VidA 2011, 397-455; Tóth et al. 2016; Vida 2018, 40-156. 


\section{Fragestellung und Ziele}

Im Fokus der Dissertation stand die Untersuche der Dynamik und der Intensität der „byzantinischen"/ mediterranen Kontakte des awarenzeitlichen Karpatenbeckens. Es bat eine Station in der Reihe, die mit der Frage der möglichen Vermittlungsprozesse, mit der Frage der Richtungen der Kontakte und der Adaptation der fremden Güter sich befasst. Das untersuchte Fundmaterial bestand in erster Linie aus den metallenen Schmuckstücken und Kleidungsbestandteilen zwischen letzten Drittel des 6. - Ende 7. Jahrhunderts aus dem Karpatenbecken.

Ein Hauptziel der Arbeit war die Erklärung und Beschreibung des Begriffs „byzantinisch“, da es viele Fragen und Problematik umfasst. Es wurde in der heimischen Forschung eher als Sammelbegriff für die aus dem Mediterraneum stammenden immateriellen (Ideen- und Wissenstransfer: z.B. Ornamentik, herstellungstechnische Verfahren, geistige Kultur: z.B. Ikonographie auf den Scheibenfibeln) und materiellen Kultur (Importwaren: z.B. Schmuckstücke, Kleidungsbestandteile) benutzt, obwohl es geographische und chronologische bzw. archäologische Problemen ebenso beinhaltet. ${ }^{8}$

Das Ziel der Dissertation ist, die „byzantinischen“ Kontakte zu thematisieren und wo es möglich ist, die Richtungen und die Art der Kontakte zu beschreiben, bzw. die Dynamik und Intensität der Kontakte innerhalb des Arbeitsgebietes zu untersuchen und zu bewerten. Den Kern der Arbeit bietet die Beschreibung der untersuchten Gegenstände, wo die Frage der Produktion, der Verbreitung und der möglichen Vermittlungsprozesse erarbeitet sind.

Ziel wurde weiterhin ausgerichtet, der archäologische Niederschlag der untersuchten Funde im Arbeitsgebiet und ihre Schwankungen in chronologischer als auch in geographischer Hinsicht zu untersuchen. Unterschiedliche Adaptationsmechanismen und Imitationsprozesse wurden in der untersuchten Zeitperiode erfolgt, was in der Menge der Gegenstände und in der Art der Imitation und Adaptation ebenso widerspiegelt.

In der Analyse werden außer der Gürtelgarnituren vor allem diejenigen Schmuckstücken und Kleidungszubehör hervorgehoben, die in relativ größerer Zahl vorkommen, und ihre Verbreitung relativ gut dargestellt werden kann. Als „Kontrollgruppe“ wurden diejenige Artefakte genommen, die bestimmt nicht Ort gefertigt wurden (Amphoren, Textilien, Edel- und Buntemetallgefäße, Möbel, Elfenbein, Mozaiksteine, Gläser usw.) daher als Import genannt werden konnten.

\section{Quellengrundlage}

Da ein Hauptziel der Dissertation war, nach Parallelen aus dem ganzen Mediterraneum zu suchen, man soll die Grenzen dieser Forschung erwähnen, weil der Forschungsstand der byzantinischen Artefakte im Mittelmeerraum unterschiedlich ist. Wichtiger Ausgangspunkt ist der Publikationsstand aus rein quantitativem Aspekt, da die Zahl der Publikationen hinter dem aus West- und Mitteleuropa bekannten Niveau bleibt. ${ }^{9}$ In den Mitteilungen wurde lange die Darstellung von Großbaustrukturen bevorzugt, ${ }^{10}$ und bei den Ausgrabungen waren die Schichten aus den byzantinischen Perioden am meisten vernachlässigt. Die materielle Kultur war lange anhand in den Sammlungen gelangten Objekten bekannt, die aber ohne Kontext

8 BolLóK 2015, 265-314.

9 Riemer 1992, 7-15; Drauschie 2011, 22.

10 Mango 1986; WoŁoszyn 2006, 262. 
standen. Ausnahme bedeutete die Keramik, die Veränderungen begonnen mit derer Untersuche ${ }^{11}$ was seitdem viel fortgeschritten hat. ${ }^{12}$ In anderen Bereichen der materiellen Kultur wurden aber seitdem mehrere wissenschaftlichen Bearbeitungen durchgeführt. Innerhalb der Goldschmiedearbeiten wurden schon mehrere Typen behandelt, ${ }^{13}$ und das technologische „know how" wurde ebenso untersucht. ${ }^{14}$ Vor allem die Goldschmiedearbeiten der mittel- und spätbyzantinischen Zeit wurden systematischer betrachtet,,$^{15}$ gegenüber den frühbyzantinischen Stücken, ${ }^{16}$ da der Übergang zwischen der spätantiken und frühbyzantinischen Zeit unscharf ist. Fortschritte wurden immerhin in der byzantinischen Glaskunst ${ }^{17}$ oder in der Textilforschung ${ }^{18}$ ebenso erzielt. Wesentlich hat sich die Forschung des Bereiches des alltäglichen Lebens, wie z.B. materielle Kultur, Literatur, Magie oder Sitte ${ }^{19}$ - entwickelt.

Regionale Unterschiede lassen sich in der Publikationsstand beobachten. Solange in Italien oder auf der nördlichen Seite des Balkangebietes mehrere Gräberfelder schon publiziert sind und die typologische Untersuche der Artefakte wurde auch fortgeschritten, die Anatolien ist immer noch ein weißer Fleck auf der Karte hinsichtlich der Forschung der byzantinischen Kleinfunde. Die Verbreitung mehreren Gegenstandtypen im Arbeitsgebiet haben Parallelen aus dem Pontus-Gebiet und keine aus dem Mittelmeerraum. Die Möglichkeit würde sich anbieten, dass solche Typen aus Anatolien vorkommen, das bleibt aber die Arbeit der zukünftigen Forschung.

"byzantinisch"
\begin{tabular}{|c|l|l|l|}
\hline Identifizierungsmodelle & Quast 2001 & Daim 2000 & $\begin{array}{c}\text { Herinrich- } \\
\text { Tamáska 2005 }\end{array}$ \\
\hline Inschriften & & & \\
\hline $\begin{array}{c}\text { Ausverstung der } \\
\text { Verbreitungskarten }\end{array}$ & & & \\
\hline Metallanalysen/Material & & & \\
\hline Herstellungstechnik & & & \\
\hline Stil & & & \\
\hline Form & & & \\
\hline Verzierung & & & \\
\hline Motiv & & & \\
\hline Funktion & & & \\
\hline
\end{tabular}

Abb. 1. Identifizierungsmodelle für die Frage „was ist byzantinisch”.

11 HAYES 1972.

12 Spieser 1996; Böhlendorf-Arslan 2004; BöHLEndorf-Arslan et al. 2007; Böhlendorf-Arslan 2017.

13 D’Angela 1989; Yeroulanou 1999; Baldini Lippolis 1999; Riemer 2000; DrauschKe 2010, 175-188; BosselMANN-RUICKBIE 2011.

14 Bosselmann-Ruickiie 2014, 333-369.

15 Bosselmann-Ruickiıe 2008, 83-114; Albani 2010, 193-202.

16 Die Hintergrund dafür kann man in der Problematik der Übergang zwischen spätantiken und frühbyzantinischen Zeit benennen.

17 DrauschKe - Keller 2010.

18 LiNSCHEID 2011; LINSCHEID 2016, 837-841.

19 Kukules 1948; Spier 1993, 25-62; Schreiner 2001; Papanikola-Bakirtzis 2002; Bollók 2013, 227-241; BosSELMANN-RUICKBIE 2017, 293-307. 


\section{Theoretischen Grundlage}

Die theoretischen Rahmen der Dissertation berühren mehrere Aspekte, dabei die Frage der Identifizierung der Artefakte fremder Herkunft einen wesentlichen Element bildet. Hauptfrage ist, ob das Artefakt im Ort gemacht wurde, und danach wer hat es gefertigt? Wenn es fern hergestellt wurde, geht es tatsächlich um Import (im Sinne inportare) oder Mobilität, aber dann tauchen weiterhin Fragen über die Vermittlung auf. Obwohl es möglich ist, dass ein Artefakt lokal gefertigt wurde, ist es aber jedoch Produkt eines fremden Handwerkers oder Goldschmiedes. ${ }^{20}$ Innerhalb dieser Problematik ist die Frage ,byzantinisch" und ,mediterran ein spezielles Themenfeld. Die unterschiedlichen Identifizierungsmodelle ( $A b b$. 1), bzw. die Grenzen der Verwendbarkeit des Begriffes „byzantinisch“ werden hier vorgelegt. Es wurde festgelegt, dass das untersuchte Material in erster Linie nach der geographischen Verbreitung sortiert wurde, danach wurden die Ornamentalen und herstellungstechnischen Aspekte in Betracht gezogen (Abb. 2).

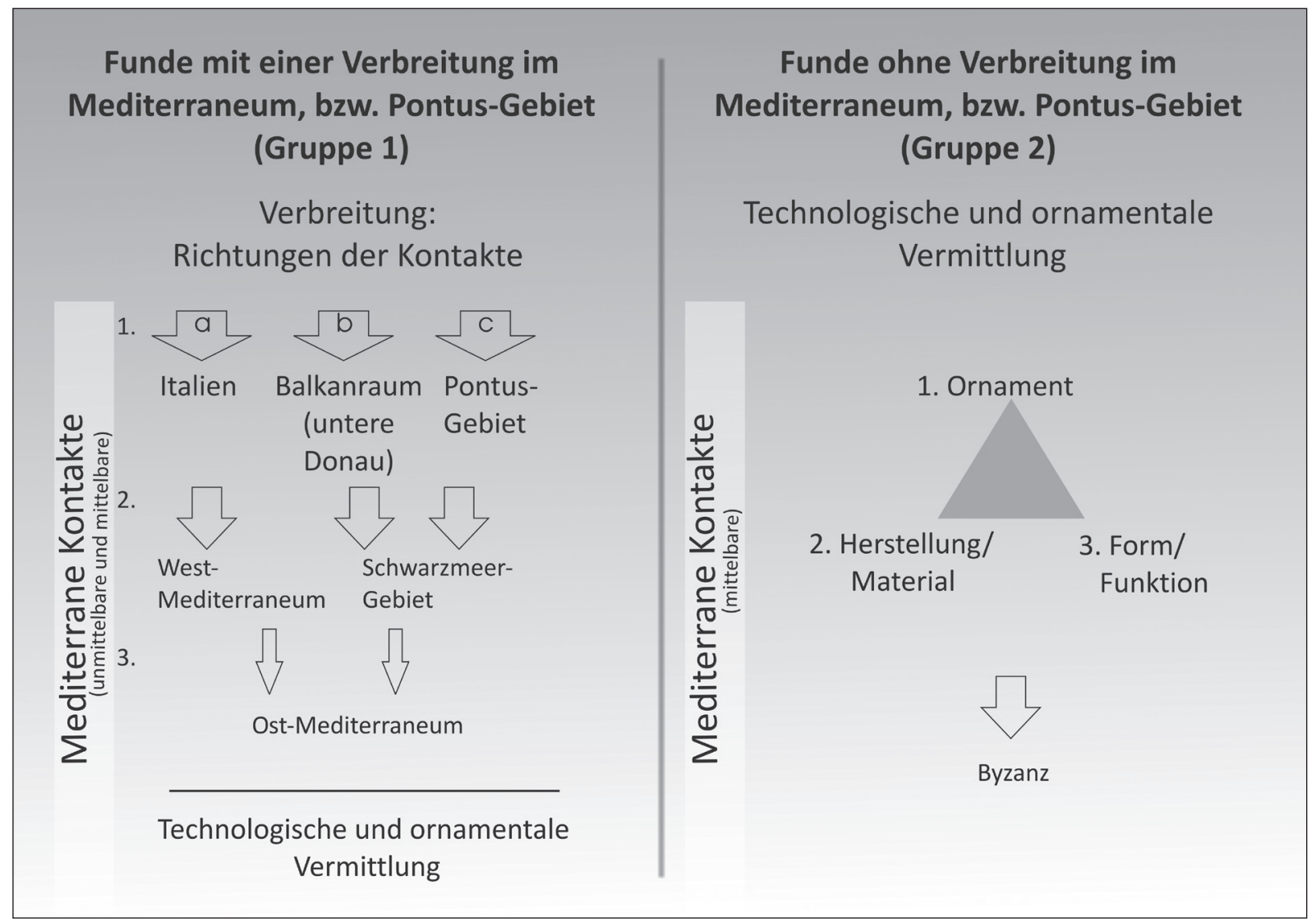

Abb. 2. Geographische Gliederung der mediterranen Kontakte.

Nach der Erörterung dieses Thema wurden die Möglichkeiten der Beziehungen zwischen zwei Kulturen und die Kanäle der Übermittlung bzw. die die Interpretierungsmöglichkeiten der Artefakte im Grab behandelt werden. Diese Fragekreise hängen eng zusammen, da der kulturelle Hintergrund einer Gesellschaft stark die Art der Kontakte beeinflusst und hat Auswirkungen ebenso auf die Kanäle der Übermittlung. ${ }^{21}$

21 UlF 2014b, 507-566. 
Der Leitfaden der Analyse der Kulturkontakte beinhaltet drei Fragen:

- Welche Formen von Interaktionen zwischen Einzelpersonen, Gruppen und Gesellschaften lassen sich anhand der verfügbaren Quellen nachvollziehen und welche Kriterien erlauben eine Differenzierung dieser Interaktionsformen?

- Welche wechselseitigen Auswirkungen hatten diese Interaktionen und Fernkontakte auf verschiedene soziale Gruppen und deren Strukturen (z. B. Selbstdarstellung bzw. Legitimation von Eliten, Breitenwirkung) sowie auf die Veränderung von Identitäten? Inwieweit lassen sie sich mit archäologischen Methoden erfassen?

- Inwieweit wurden durch den Austausch von Objekten auch immanente fremde Ideen adaptiert oder erfuhren eine Umdeutung ihrer gesellschaftlich-sozialen Funktion und Bedeutung in dem neuen, fremden Kontext? ${ }^{22}$

Da es in den meisten Fällen schwierig ist die Vermittlungsprozesse der Gegenstände zu entscheiden, könnten jedoch in einigen Fällen näher begrenzen, ob es über Diplomatie, Geschenk, Handel, Beute, Mobilität geht. Die Bewegung der Güter hängt natürlich mit der Mobilität der Personen zusammen, darauf aber, wie sie vermittelt wurden, kann man nur folgern.

\section{Ergebnisse}

Im Karpatenbecken wurden 22 Schmucktypen und 12 Kleidungsbestandteiltypen mediterraner Herkunft untersucht, die insgesamt 1571 Stücke bedeuten.

1. In der zeitstufenmäßigen Analyse konnte festgestellt werden, dass am Ende 6. - Anfang 7. Jahrhunderts das Spektrum der Schmuckstücke und Kleidungszubehör vielfältiger ist, als in den folgenden Zeithorizonten. Mehrere Typen sind in kleinerer Menge bekannt. Die meisten Schmuckstücke, die am Ende 6. - Anfang 7. Jahrhunderts verwendet waren, sind in der I. Hälfte des 7. Jahrhunderts auch noch ins Grab gelegt. Die Menge ist abwechslungsreich, es gibt Kleidungszubehöre, die nur in geringer Zahl (1 oder 2 Stück) vorkommen, es gibt aber solche auch, die schon in einer größeren Menge auftreten. Gegenüber dem früheren Horizont kann in der II. Hälfte des 7. Jahrhunderts festgestellt werden, dass es sich um weniger Typen handelt, die aber in größerer Menge vorkommen. Sie sind in größerer Zahl nachgeahmt und massenhaft produziert, wie z.B. die Ohrringe mit kleinen Granulationen und die Ohrringe mit aufgezogener Blechkugel. In der II. Hälfte 7. Jahrhunderts kommen die früheren Typen noch sporadisch vor - zwar etwas umgeformt, aber weiterleben - und neuere Typen häufen sich an. Sie kommen schon am Ende des 7. Jahrhunderts auch in größerer Zahl vor, es gibt aber sehr viele, die undatierbar sind (Abb. 3).

2. Während des 7. Jhs. blieben alle drei Richtungen - nach Italien, nach Balkan und nach Pontus - wichtig und maßgeblich. Die Kontakte mit Italien waren in der I. H. 7. Jhs. lebendiger, wurden aber in der II. H. 7. Jhs. auch nicht aufgehört. Das Transdanubien blieb mit dem Ostalpenraum ebenso in Verbindung. Die balkanischen Beziehungen waren am Ende 6. - Anfang 7. Jhs. bedeutend, mehrere Artefakte stammen aus diesem Gebiet oder reichten das Karpatenbecken aus dieser Richtung. Die Verbindung mit dem Schwarzmeergebiet kann unter dem ganzen 7. Jh. beobachtet werden, ihre Bedeutung wächst aber in der II. H. 7. Jhs. Obwohl es in diesem Fall sich 

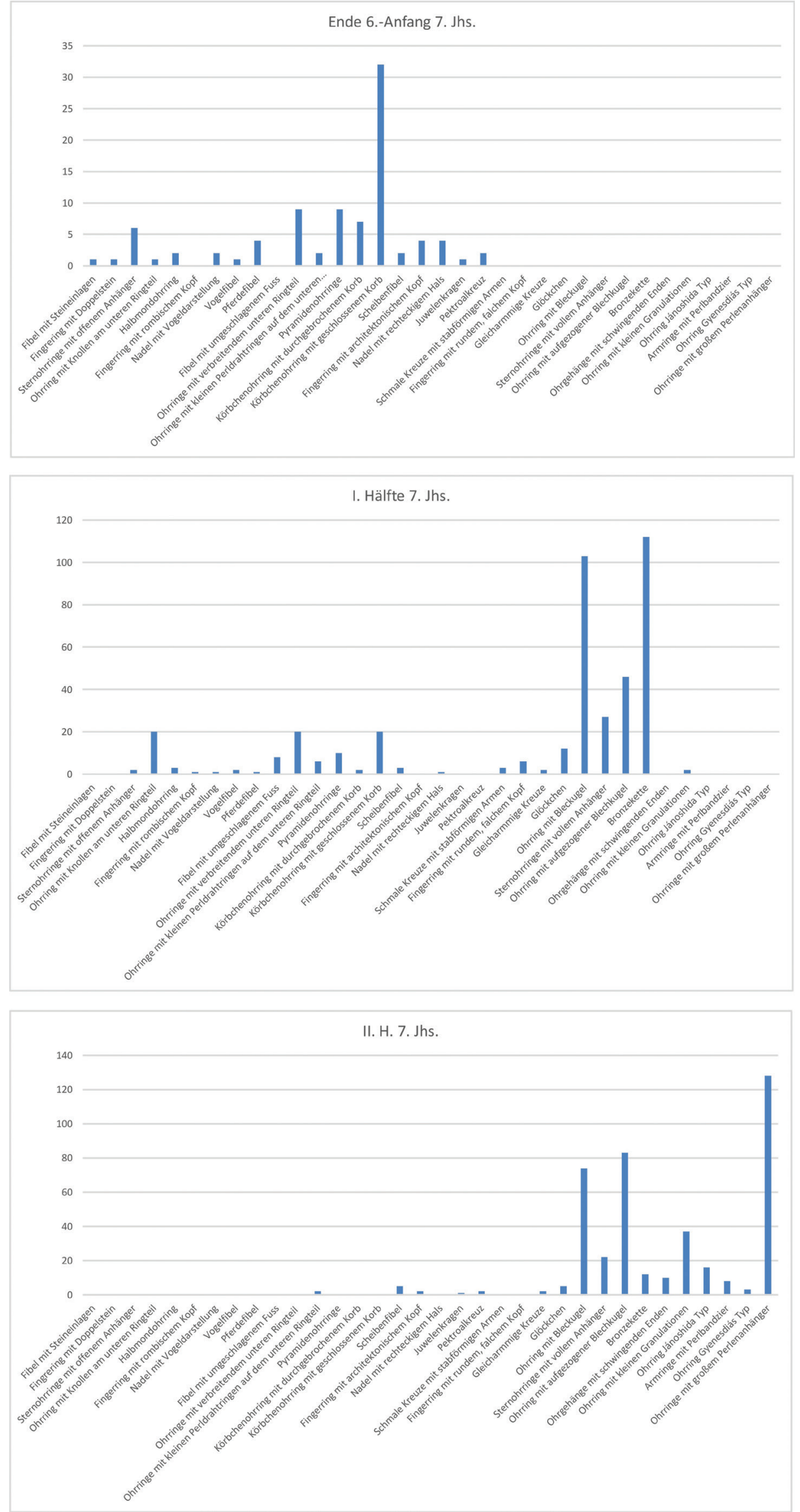

$A b b$. 3. Die zeitliche Verteilung der untersuchten Gegenstände. 
voneinander weit entfernten Regionen handelt, manche Gegenstände zeigen, dass das Karpatenbecken, das Schwarzmeerraum und das Gebiet nördlich vom Schwarzen Meer zu einem Kommunikationsraum gehörte. Die Intensität der Kontakte und die Zahl der Fundtypen steigen sich etwas bis der Ende der I. Hälfte 7. Jahrhunderts, die Verwendung einzelnen Typen endet sich aber bis der Jahrhundertmitte. Einige Artefakte exzeptioneller Qualität sind aus dem früheren Fundmaterial herausragend. Ab I. H. 7. Jahrhundert könnten die italienischen bzw. westmediterranen Kontakte eines Teiles der Bevölkerung (vor allem in Süd- Transdanubien) stark sein, sie waren eng miteinander vernetz, so dass die Waren-, Ideen-, und Technologietransfer relativ schnell durchgesetzt werden konnte. (Abb. 4).

3. Die Untersuche in der Aktivität der unterschiedlichen Regionen hat gezeigt, dass in der der früheren Periode vor allem die ehemalige Pannonien und die südlichen Regionen der Donau-Theiß Zwischenstromgebiet bzw. die südliche Tiefebene größere Rolle bekommen. In der Frühphase zeigen diese Regionen eine gewisse Aktivität hinsichtlich der mediterranen Kontakte auf. Ab des 2. Drittels, bzw. ab der Mitte des 7. Jahrhunderts nimmt die Wichtigkeit dieser Regionen ab, die Intensität und Dynamik der Kontakte der Bevölkerung dieser Regionen in die Richtung Mediterraneums verändert und der Schwerpunkt der mediterranen Kontakte verschiebt sich nach Norden (Abb. 5).
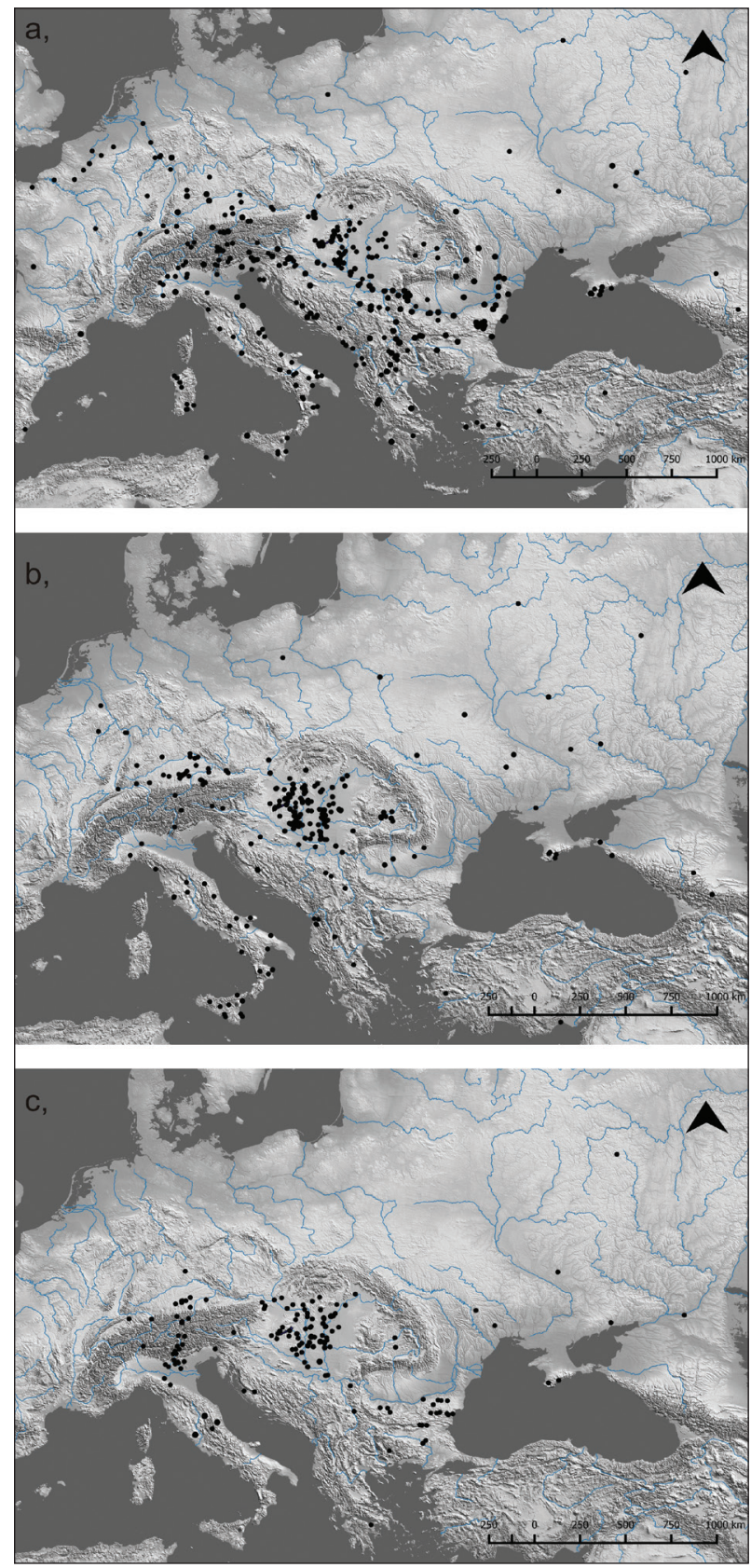

$A b b$. 4. Kontakte der untersuchten Gegenstände: a - am Ende 6. - Anfang 7. Jhs., b - erste H. 7. Jhs., c - II. H. 7 Jhs.

Wichtig ist die Region um Szeged in der I. 7. Jhs. Zwar kommen nicht so viele Schmucktypen und Kleidungszubehörtypen in dieser Zeit hier vor, es gibt jedoch einige Gräberfelder, die wichtige Rolle in den mediterranen Kontakten gespielt haben könnten, die aber in der Forschung noch nicht weit bekannt sind. Beispielsweise im Gräberfeld von Makó-Mikócsa-halom wurde neben einem Goldkreuz z.B. ein aus Elfenbein gefertigter Gegenstand vorgekommen. Der Donau-Theiss Zwischenstromgebiet bekommt ab der II. H. 7. Jhs/dritten Drittel des 7. Jhs. größere Rolle, wenn die reich ausgestatteten Elitengräber hier erscheinen. Die Mehrheit der Gegenstände mediterraner Herkunft konzentriert sich in diesen Bestattungen.

Transsylvanien spielte in der Awarenzeit eine spezielle Rolle. Es wurde letztlich als Peripherie behandelt, gleichzeitig lassen sich aber solche Phänomene hier beobachtet werden, die 
in Transdanubien vorgekommen wurden, wie z.B. ähnliche Schmuckstücke und Kleidungszubehör in der Frühphase auftauchen. Diese Ähnlichkeit dafür erklärt die frühere römische Tradition in beiden Regionen.

Während der Untersuchung wurde klargeworden, dass solange in der I. H. 7. Jhs. vor allem Pannonien, die Region um Balaton und Süd-Transdanubien sehr aktiv in den mediterranen Beziehungen war, in der II. 7. Jhs. sind die nördlichen Regionen des Donau-Theiss Zwischenstromgebiets und der Thießregion viel mehr betroffen. Die Aktivität der Regionen in der ersten Jarhunderthälfte hört nicht auf. Sogar in diesen Gebieten (z.B. in Romonya) lässt sich das Weiterleben einzelnen Artefakten aus der I. H. Jhs. feststellen.

Was die Richtungen der Kontakte trifft, sie konnten zusammenfassend in zwei Gruppen geteilt werden. $\mathrm{Zu}$ der ersten Gruppe (1.) gehören also diejenigen Artefakte, die direkte Parallelen im Mediterraneum haben. Die Gruppe 2 steht für diejenigen Artefakten, die keine Parallelen im Mediterraneum haben, ihrer Form, Technologie und Verzierung nach in der Forschung jedoch „byzantinisch" bestimmt werden (Abb. 2).

4. Als Auswirkung der mediterranen Kontakte soll der Aufhören der Interaktionen ebenso betrachten, was während der II. 7. Jhs. eine veränderte Repräsentation verursachen könnte. In den schriftlichen Quellen referiert mindestens ein Quellenangabe über den diplomatischen Kontakten zwischen den
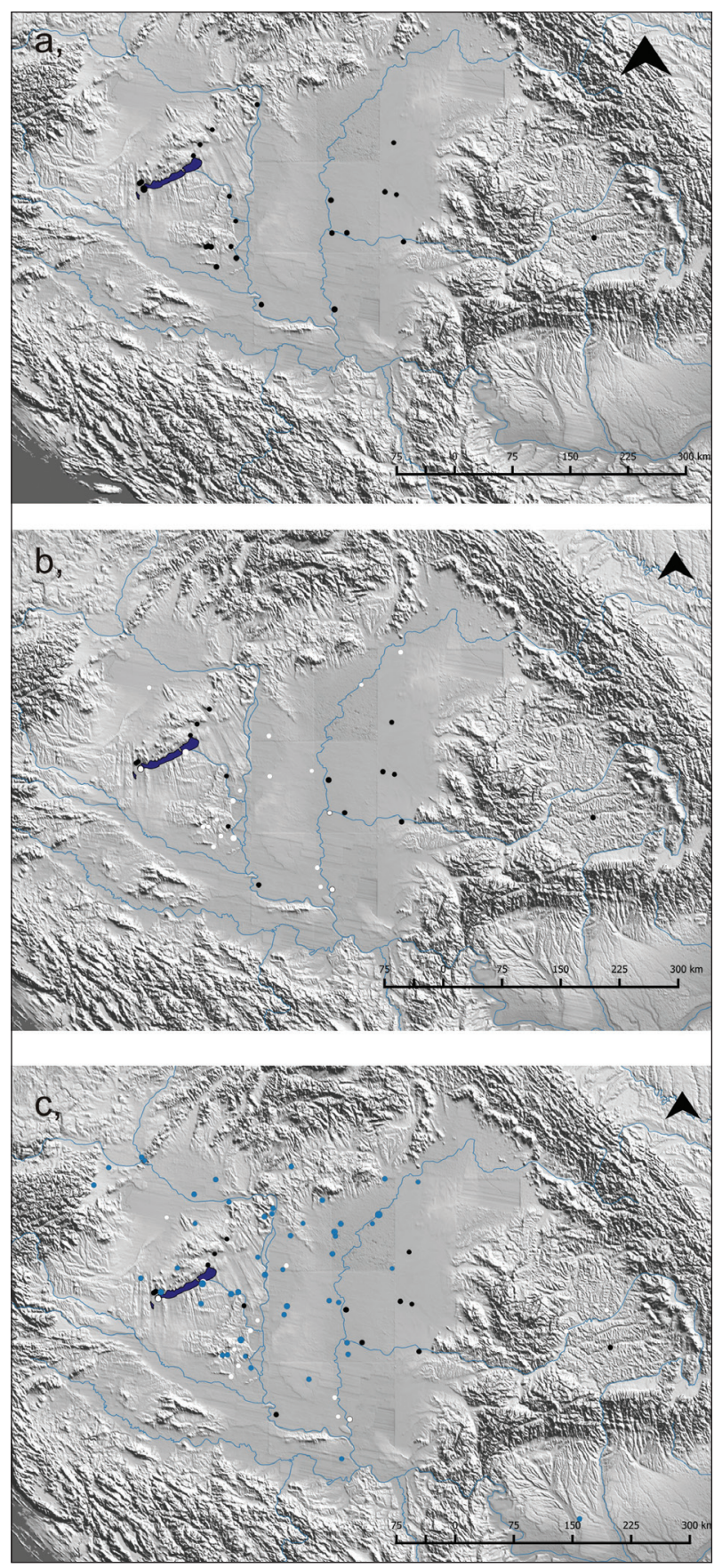

$A b b$. 5. Die aktive Regionen in der Verteilung der mediterranen Güter im Karpatenbecken: a - Ende 6. - Anfang 7. Jhs., b - erste H. 7. Jhs., c - II. H. 7. Jhs. Awaren und Byzanz in dieser Zeit, bzw. der Münzverkehr wurde für eine Periode (zwischen 626 und 650) unterbrochen. Während des 7. Jhs. hatte sich das Territorium des Byzantinischen Reiches ebenso deutlich verkleinert. Es war also eine solche Periode, in dem die Situation der Elite verändert werden konnte. Der intensive Ausdruck des Vermögens und der sozialen Status war nicht dann nötig, wenn die Eliten stabil waren, sondern dann, wenn irgendwelche Veränderungen in der Gesellschaft eingetroffen wurden. Diese Ereignisse benötigten die Demonstrierung der Macht der Elite. Das wäre eine Erklärung für die konzentriertere Deponierung der Gegenstände mediterraner Herkunft in dieser Periode. 


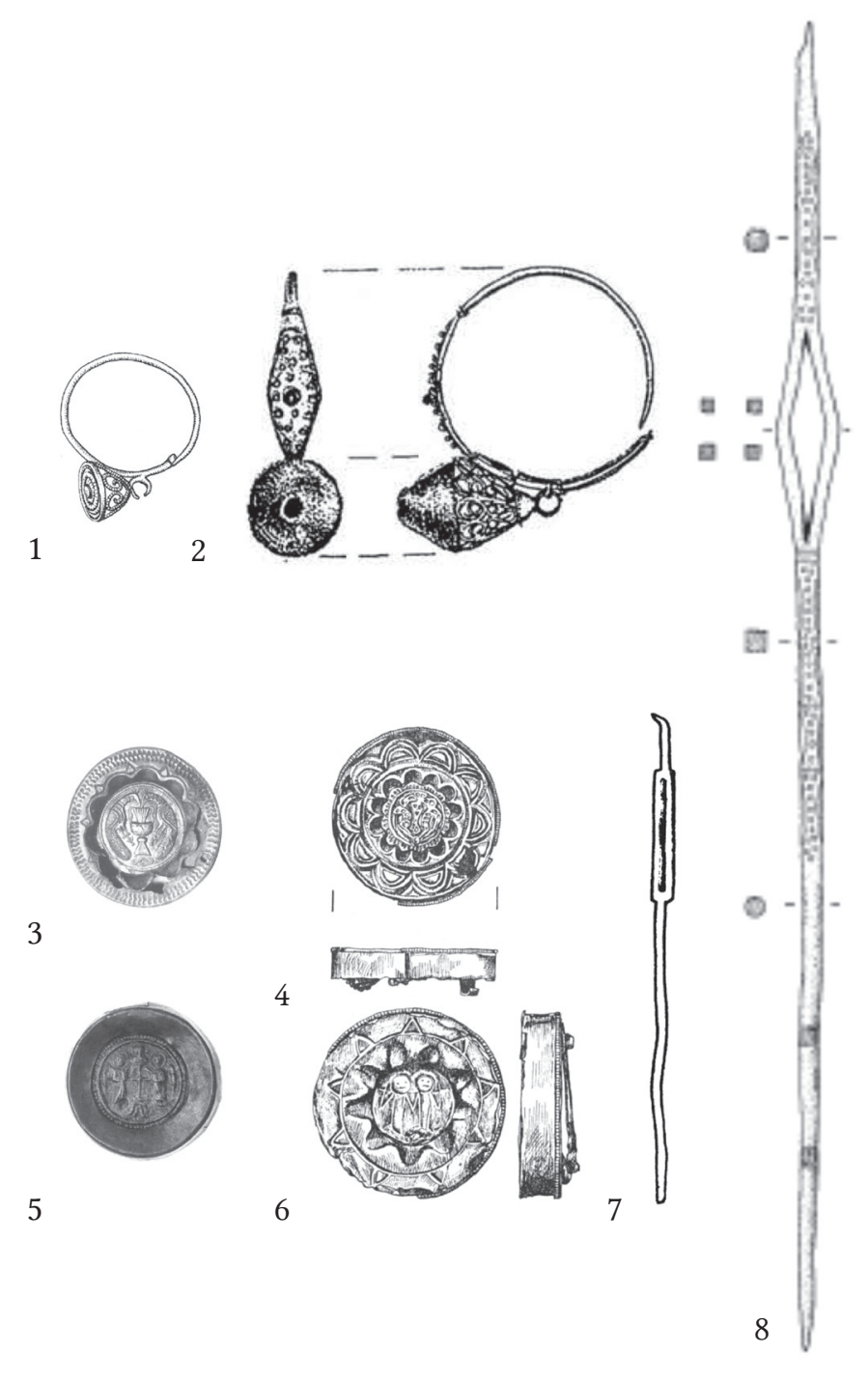

$A b b$. 6. Vergrößerung der Artefakte wärend des 7. Jhs.
Dabei konnte festgestellt werden, dass in der I. H. 7. Jhs. hauptsächlich bei den mit Presstechnik gefertigten Schmuckstücken eine Umwandlungsprozess beobachtet werden kann, solange in der II. H. 7. Jhs. die einzelnen Typen nachgeahmt und massenhaft produziert wurden.

5. In der sozialen Untersuche der Gegenstände mediterraner Herkunft konnte festgelegt werden, dass in der mediterran-byzantinischen Kontakten nicht nur wertvolle, aus Gold gefertigte Artefakte vermittelt wurden. Die Artefakte haben durch unterschiedliche Wege das Karpatenbecken erreicht. In den schriftlichen Quellen sind hauptsächlich die diplomatischen Kontakte benannt, durch denen vor allem prachtvolle Gegenstände und Luxusgüter vermittelt wurden, dem einige Gegenstände im Fundmaterial entspricht. Es gibt aber eine Reihe von Gegenstände (Tierfibeln, Nadel mit Vogelkopf, Fibel mit umgeschlagenem Fuß), die in den Gräber der weniger wohlhabenden Personen erscheinen.

Die prachtvollste Waren treten eher eine Zeitperiode später, in der II. H. 7/Mitte 7. Jhs. auf, da in dieser Zeitperiode die Intensität der diplomatischen Beziehungen zwischen den Awaren und Byzanz anhand den schriftlichen Quellen deutlich abgenommen hat, was Wirkung auf die Gesellschaft geübt haben könnte. Teil der Veränderung sein könnte, dass die Elite ihre Macht und ihren Status durch den prachtvollen, reichen Gegenstände demonstriert haben sollten. Das bedeutet aber nicht, dass die durch den diplomatischen Kontakte erworbene Gegenstände in der I. H. 7. Jhs. oder früher nicht eingeströmt waren, sind sie aber im Gräberfeldmaterial weniger sichtbar. Die Mobilität war durch mehrere Wege nach dem Mittelmeerraum möglich, bzw. der entstandene Herrschaft der Awaren und ihre Bedarf konnten die Goldschmiede von entfernten Regionen z.B. aus dem Balkan ins Karpatenbecken angezogen.

6. In der Untersuche wurde weiterhin klar geworden, dass es einige Artefakte gibt, die ganz langlebig sind, und in der Zeit wurden sie in immer größeren Form hergestellt ( $A b b$. 6). Diese Artefakte kann man vor allem mit der Gesellschaften um Balaton und in Südpannonien in Verbindung bringen. Dieses Phänomen ist aber nicht einzigartig und ist nicht nur eine lokale Erscheinung Die Vergrößerung der Körbchenohrringe ${ }^{23}$ kann man in den bayerischen 
Gebieten ebenso beobachten. Der Grund für diesen Vergrößerungsprozess bedarf noch weitere Forschungen in dieser Zeitperiode.

Anhand den untersuchten Fundmaterial konnte man feststellen, dass die Macht und Herrschaft der Awaren ein Teil der umgebenden Welt bedeuteten. Vor allem die Elite aber damit zusammenhängend mittelbar die ganze Bevölkerung war von den äußeren Veränderungen betroffen. Neben den eigenen Bedürfnissen spiegelt das Fundmaterial des Arbeitsgebietes die Veränderungen im Mediterraneum und in den breiteren Gebieten ebenso wider.

\section{Literaturhinweise}

Albani, J. 2010: Elegance over the Borders: The Evidence of Middle Byzantine Earrings. In: Entwistle, C.-Adams, N. (eds): Intelligible Beauty. Recent Research on Byzantine Jewellery. British Museum Research Publication 178. London,193-202.

BALDINI Lippolis, I. 1999: L'oreficeria nell'lmpero di Costantinopoli tra IV e VII Secolo. Bari Edipuglia.

BAUER, A. 2008: Import, Imitation or Communication? In: Biel, P. F.-RAssAmakin, Y. YA. (eds): Import and Imitation in Archaeology. Schriften des Zentrums für Archäologie und Kulturgeschichte des Schwarzmeerraumes. Langenweißbach, 98-104.

BolLóK, Á. 2013: Apotropaion and burial in Early Byzantium. Some preliminary considerations. In: JuHÁsz, E. (ed.): Byzanz und das Abendland: Begegnungen zwischen Ost und West. Budapest, 227-241.

Bollók, Á. 2015: The Archaeology of the Byzantine State - A Non-Specialist's Approach. Antaeus 33, 265-314.

Bosselmann-Ruickbie, A. 2008: Byzantinisch, Islamisch oder „Internationaler Stil“? Email- und Körbchenohrringe aus dem östlichen Mittelmeerraum. In: Koenen, U.-MülLER-Wiener, M. (eds): Grenzgänge im östlichen Mittelmeerraum. Byzanz und die islamische Welt vom9.bis 13. Jahrhundert, Wiesbaden, 83-114.

Bosselmann-RuickiIe, A. 2011: Byzantinischer Schmuck des 9. bis frühen 13. Fahrhunderts. Wiesbaden 2011. doi: $10.29091 / 9783954908547$

Bosselmann-Ruickbie, A. 2014: Antje Bosselmann-Ruickbie: Das Verhältnis der ,Schedula diversarum artium' des Theophilus Presbyter zu byzantinischen Goldschmiedearbeiten: Grenzüberschreitende Wissensverbreitung im Mittelalter? In: SPEER, A. (Hrsg.): Zwischen Kunsthandwerk und Kunst: Die „Schedula diversarum artium”. Berlin-Boston, 333-369.

BöHLendorf-Arslan, B. 2004: Glasierte byzantinische Keramik aus der Türkei. Istanbul.

BöHLENDORF-Arslan, B. 2017: Schiffsdarstellungen auf spätantiker und byzantinischer Keramik, In: Friehlinghaus, E.-Schmidts, T.-Tsamakda, V.: Schiffe und ihr Kontext: Darstellungen, Modelle, Bestandteile von Griechenland bis Byzanz. Internationale Tagung Mainz 24.-25. Mai 2013. Mainz, 173-183.

Böhlendorf-Arslan, B. - Uysal, A.O. - Witte-Orr, J. 2007: Late Antique and Medieval Pottery and Tiles in Mediterranean Archaeological Contexts. Proceedings of the First International Symposium on Late Antique, Byzantine, Seljuk, and Ottoman Pottery and Tiles in Archaeological Context. BYZAS. Veröffentlichungen des Deutschen Archäologischen Instituts Istanbul 7, 399-410.

Csiky, G. 2015: Avar-age Polearms and Edged Weapons. Classification, Typology, Chronology and Technology. East Central and Eastern Europe in the Middle Ages, 450-1450. 32. Leiden. doi: 10.1163/9789004304543

Csiky, G. - Magyar-HÁrshegyi, P. 2015: Wine for the Avar elite? Amphorae from Avar period burials in the Carpathian Basin, in: Tsetsknladze, G. R.-Avram, A.-Hargrave, J. (eds): The Danubian Lands between the Black, Aegean and Adriatic Seas. $\left(7^{\text {th }}\right.$ century BC $-10^{\text {th }}$ century AD) Proceedings 
of the Fifth International Congress on Black Sea Antiquities (Belgrade - 17-21 September 2013). Oxford, 175-182. doi: 10.2307/j.ctvr43k44.29

D’Angela, C. 1989: Ori Bizantini del Museo Nazionale di Taranto. Taranto.

DrauschKe, J. 2010: Halbmondförmige Goldohrringe aus bajuwarischen Frauengräbern - Überlegungen zu Parallelen und Provenienz. In: Daim, F.-Drauschke, J. (Hrsg.): Byzanz - das Römerreich im Mittelalter. Monographien des RGZM 84. Mainz, 175-188

Drauschke, J. 2011: Zwischen Handel und Geschenk - Studien zur Distribution von Objekten aus dem Orient, aus Byzanz und aus Mitteleuropa im östlichen Merowingerreich. Freiburger Beiträge zur Archäologie und Geschichte des ersten Jahrtausends 14, Rahden/Westf.

Drauschke, J. - Keller, D. 2010: Glass in Byzantium - Production, usage, analyses. Glas in Byzanz Produktion, Verwendung, Analysen. RGZM Tagungen 8, Mainz.

FeHR, H. - NowAK-Böck, B. - SöcKL, T. 2004: Eine runde Sache: Restaurierung und Ausstellung der frühmittelalterlichen Funde von Bruckmühl; Landkreis Rosenheim, Oberbayern. Das archäologische fahr in Bayern, 179-182.

Garam, É. 2001: Funde byzantinische Herkunft in der Awarenzeit vom Ende des 6. Bis zum Ende des 7. Jahrhunderts. Monumenta Avarorum Archaeologica 5, Budapest.

HajnAL, Zs. 2005: Késő antik jellegủ kerámia a Kölked-feketekapui avar kori telepről. In: Communicationes Archaeologicae Hungariae, 437-480.

Hayes, J. 1972: Late Roman Pottery. London.

Kukules, Ph. 1948: Byzantinon bios kai politismos 2. Athenai.

Linscheid, P. 2011: Frühbyzantinische textile Kopfbedeckungen. Typologie, Verbreitung, Chronologie und soziologischer Kontext nach Originalfunden. Wiesbaden. doi: 10.29091/9783954908561

Linscheid, P. 2016: Die frühbyzantinischen Textilien des Römisch-Germanischen Zentralmuseums. Mainz.

Mango, C. 1986: Byzantine Architecture. Milano-New York-London.

Papanikola-Bakirtzis, D. 2002: Everyday life in Byzantium. Athens.

RÁcz, Zs. 2014: Die Goldschmiedergräber der Awarenzeit. Monographien der Römisch-Germanischen Zentralmuseums 116, Mainz am Rhein.

RIEMER, E. 1992: Byzantinische Körbchen- und Halbmondohrringe im Römisch-Germanischen Museum Köln (Sammlung Diergardt). Kölner Jahrbuch für Vor- und Frühgeschichte 25, 121-136.

Riemer, E. 2000: Romanische Grabfunde des 5.-8. Jahrhunderts in Italien. International Archäologie 57, Rahden.

Schreiner, P. 2001: Stadt und Gesetz - Dorf und Brauch. Versuch einer histrosichen Volkskunde von Byzanz: Methoden, Quellen, Gegenstände, Beispiele. Nachrichten der Akademie der Wissenschaften zu Göttingen, I. Philologisch-historische Klasse, 569-662.

SPIESER, J.-M. 1996: Die byzantinische Keramik aus der Stadtgrabung von Pergamon. Berlin-New-York.

SpIER, J. 1993: Medieval Byzantine Magical Amulets and Their Tradition. Journal of the Warburg and Courtauld Institutes 56, 25-62. doi: 10.2307/751363

TовіAs, B. 2008: Frühmittelalterliche Gräber mit Beigabe von Schmiedewerkzeugen. Dissertation, Uniwersität Wien.

Tóth, E. - VIDA, T. - TAKÁcs, M. (eds) 2016: Saint Martin and Pannonia: christianity on the frontiers of the roman world: exhibition catalogue: Abbey Museum, Pannonhalma, 3 fune - 18 September 2016: Iseum Savariense, Szombathely, 3 Fune - 13 November 2016. Pannonhalma.

Ulf, Chr. 2014: Rethinking Cultural Contacts. In: Rollinger, R.-Schnegg, K. (Hrsg.): Kulturkontakte in Antiken Welten: Vom Denkmodell zum Fallbeispiel. Proceedings des internationalen Kolloquiums aus Anlass des 60. Geburtstages von Christoph Ulf, Innsbruck, 26. bis 30. Januar 2009. Leuven-Paris-Walpole, MA, 507-566. 
VIDA, T. 1999: Die awarenzeitliche Keramik I. Früh- und Mittelawarenzeit, 6./7. Jh., Varia Archaeologica Hungarica 8. Berlin-Budapest.

VIDA, T. 2009: Local and Foreign Romans? The Problem of the Late Antique Population of the 6th-7th Centuries AD in Pannonia. In: QUAST, D (Hrsg.): Foreigners in Early medieval Europe: Thirtheen International Studies on Early Medieval Mobility. Mainz, 233-260.

VIDA, T. 2011: Das Gräberfeld neben dem Horreum in der Innenbefestigung von Keszthely-Fenékpuszta. Mit Beiträgen von Adrien Pásztor: Auswertung der Perlen aus dem Gräberfeld Keszthely-Fenékpuszta, Horreum. In: Heinrich-Tamáska, O. (Hrsg.): Keszthely-Fenékpuszta im Kontext spätantiker Kontinuitätforschung zwischen Noricum und Moesia. Budapest-Leipzig, 397-455.

VIDA, T. 2017: Die frühbyzantinische Messingkanne mit fagdszenen von Budakalász (Ungarn). Budapest.

VIDA, T. 2018: A sztyeppei, a Bizánci és a Meroving birodalmak között. Kulturális változások a Kárpát-medence nyugati felén a 6-7. században. Academic doctoral dissertation. Budapest.

WoŁoszyn, M. 2006: Byzantine archaeology - selected problems. Analecta Archaeologica Ressoviensia 1, 259-292.

Yeroulanou, A. 1999: Diatrita. Athen. 